\title{
Aspirin in the secondary prevention of unprovoked thromboembolism: the WARFASA and ASPIRE studies
}

\author{
Simone Birocchi $\cdot$ Emanuela Scannella $\cdot$ \\ Laura Ferrari - Gian Marco Podda • \\ Gruppo di Autoformazione Metodologica (GrAM)
}

Received: 1 May 2013/Accepted: 13 May 2013/Published online: 7 June 2013

(C) SIMI 2013

\section{Background}

The treatment of unprovoked venous thromboembolism (VTE) is anticoagulant therapy for at least 3 months [1]. As VTE recurs frequently and about $20 \%$ of the patients with an unprovoked VTE develop recurrence within the first 2 years [2], extended vitamin $\mathrm{K}$ antagonist (VKA) treatment is often considered. The decision to prolong VKA therapy after the initial treatment period is a dilemma, since longer term therapy reduces the risk of recurrent thromboembolism, but increases the risk of bleeding. The last American College of Chest Physicians (ACCP) EvidenceBased Clinical guidelines suggest a strategy to balance the benefits and risks of different durations of anticoagulant therapy; in unprovoked VTE, ACCP guidelines suggest extended anticoagulant therapy if bleeding risk is low or moderate (Grade 2B) and recommend 3 months of therapy if bleeding risk is high. When extended anticoagulant therapy is contraindicated, aspirin might be appealing. In fact the annual risk of major bleeding is only $0.1 \%$ in patients on long-term low-dose aspirin therapy, thus lower than VKA therapy [3]. A large meta-analysis by the Antiplatelet Trialists' Collaboration shows that antiplatelet therapy significantly reduces the risk of fatal or non-fatal pulmonary embolism (PE) by $25 \%$ [3]. Moreover, a large trial involving patients undergoing surgery for hip fracture shows a $36 \%$ risk reduction in VTE in the aspirin therapy

S. Birocchi · L. Ferrari · G. M. Podda

U. O. Medicina III, Ospedale San Paolo,

Università degli Studi di Milano, Milan, Italy

E. Scannella $(\square)$

Dipartimento di Medicina Interna, Ospedale L. Sacco,

Università degli Studi di Milano, Milan, Italy

e-mail: emanuelascannella@gmail.com group, [4] suggesting that antiplatelet therapy may be an alternative to prolonged VKA therapy.

Two randomized controlled studies were conducted to evaluate the use of ASA for the prevention of venous thromboembolism recurrence: the Aspirin for the Prevention of Recurrent Venous Thromboembolism (Warfarin and Aspirin [WARFASA]) [5] and the Aspirin to Prevent Recurrent Venous Thromboembolism (ASPIRE) study [6].

\section{WARFASA}

\section{Summary}

The Aspirin for the Prevention of Recurrent Venous Thromboembolism (VTE) (Warfarin and Aspirin [WARFASA]) [5] is a multicentre, randomized, double-blind, event-driven clinical trial, assessing the benefit of aspirin in the secondary prevention of venous thromboembolic events after the discontinuation of VKA therapy. Patients were considered eligible if they were diagnosed with a first-ever, unprovoked (defined by the absence of any known risk factor for this event), symptomatic and instrumentally confirmed proximal deep-vein thrombosis or pulmonary embolism and if they were treated with vitamin $\mathrm{K}$ antagonists for 6-18 months. Most important exclusion criteria were known cancer, known major thrombophilia, active bleeding or high risk for bleeding or a bleeding episode, which occurred during the 6-18 months of anticoagulation. The primary outcome was the recurrence of VTE, defined as deep-vein thrombosis or pulmonary embolism. The principal safety concern outcome was major bleeding. The secondary outcomes included arterial ischemic events (myocardial infarction, unstable angina, stroke, transient ischemic attack, acute limb ischemia) and death from any cause. 
The primary efficacy analysis, which considered all outcome events occurring from randomization to the end of the study, was performed according to a modified intention-to-treat principle, with all patients who received at least one dose of the assigned study drug after randomization included in the analysis; an "on-treatment" analysis was also performed. During the study the protocol was modified: the primary outcome changed from a composite endpoint in which venous and arterial events and deaths were considered, to a simple endpoint (venous thromboembolism only).

A total of 403 patients were included; of these patients 205 were randomly assigned to aspirin $100 \mathrm{mg} / \mathrm{day}$ and 198 to placebo for 2 years of therapy. The primary outcome occurred in 5.9 and $11.0 \%$ patients year in the aspirin and placebo group, respectively, corresponding to an Hazard Ratio of 0.58 (95\% CI 0.36-0.93). A multivariate analysis adjusted for age, sex, index event (deepvein thrombosis or pulmonary embolism) and length of anticoagulant therapy confirmed the efficacy of aspirin. Two episodes of major bleeding occurred, one in each study group. There were no significant differences between the two groups in the secondary outcomes.

Strengths of the study

- It addresses a relevant clinical issue. Epidemiological evidence shows that aspirin reduces the incidence of symptomatic pulmonary embolism (PE) in subjects with or without previous episodes of VTE. There is no evidence to support a role for aspirin in the secondary prevention of VTE.

- The primary endpoint is clinically relevant.

- Two years are an appropriate follow-up period to evaluate thromboembolic events.

Weaknesses of the study

- The primary endpoint was changed during the study period, exposing the trial to the risk of bias.

\section{Question marks}

- Patients with cancer were excluded from the trial, however, four patients died from cancer and it is not specified the number of patients with a new cancer diagnosis during the study period. It would be interesting to have more data on these oncologic patients.

- Few patient characteristics were reported in Table 1. Considering also that recent guidelines [1] suggest a strategy that considers both thrombotic and hemorrhagic risk factors of individual patients to decide on the duration of anticoagulant therapy, we wonder if more data on patients could help in the understanding of which patients are likely to benefit most from aspirin therapy.

\section{Sponsorship}

It is an independent study; Bayer provided aspirin and placebo but had no other role in the study.

\section{ASPIRE}

\section{Summary}

The Aspirin to Prevent Recurrent Venous Thromboembolism (ASPIRE) study [6] was a double-blind, randomized, placebo-controlled study, evaluating the use of low-dose aspirin in preventing the recurrences of venous thromboembolism, in patients who had had a first episode of unprovoked venous thromboembolism and who had completed initial anticoagulation therapy.

Patients included were at least 18 years of age and had had a first unprovoked (i.e. in the absence of a predefined transient risk factor during the preceding 2 months) episode of objectively diagnosed symptomatic deep-vein thrombosis or an acute pulmonary embolism occurred within 2 years before enrollment. All patients were required to have completed initial anticoagulation therapy with heparin followed by warfarin (or an alternative anticoagulant) for a period between 6 weeks and 24 months. The main exclusion criteria were indications/contraindications for aspirin use, other antiplatelet therapy, or nonsteroidal anti-inflammatory drug, indications for continuing oral anticoagulation therapy, active bleeding or high risk for bleeding, life expectancy less than 12 months. The primary outcome was the recurrence of VTE, defined as deep-vein thrombosis or pulmonary embolism. The two secondary composite outcomes were major vascular events (myocardial infarction, stroke, venous thromboembolism, or cardiovascular death) and the measure of the net clinical benefit (defined as a reduction in the rate of the myocardial infarction, stroke, venous thromboembolism, major bleeding, or death from any cause). The primary safety outcome was bleeding (major bleeding or clinically relevant nonmajor bleeding).

The ASPIRE study was originally designed to recruit 3,000 patients, but the target sample size was then reduced to 1,500 patients because of slow recruitment. The authors planned to combine the final results of the study with those of the WARFASA trial [5], in a meta-analysis, with interim trial results concealed. Recruitment closed in August 2011 
because of limited resources and the study was stopped on 31 March 2012, after publication of the results of the WARFASA study [5], since the ASPIRE results could be influenced if some patients switched to aspirin on the basis of the results of the WARFASA.

The analysis for primary and secondary outcomes was performed according to an intention-to-treat model. Additional analyses were performed with adjustment for non adherence rate (the proportion of patients assigned to aspirin who discontinued it and the proportion of patients assigned to placebo who initiated antiplatelet or anticoagulation) and interactions in Cox models were used to assess differences in the effect of aspirin across prespecified subgroups (according to age, gender, duration of initial anticoagulation therapy, BMI, and type of first unprovoked event). A final meta-analysis was performed with the combined results of the ASPIRE and WARFASA [5] trials.

A total of 822 patients were randomly assigned to receive aspirin $100 \mathrm{mg} /$ day or matching placebo, for a minimum of 2 years; the maximum duration of treatment was 4 years. The median of follow-up was 37.2 months. No significant differences emerged between the two groups for the primary outcome, since VTE recurred in 73 of 411 patients assigned to placebo and in 57 of 411 assigned to aspirin (hazard ratio [HR] with aspirin, 0.74; $95 \%$ confidence interval $[\mathrm{CI}], 0.52-1.05 ; P=0.09)$. The analysis in subgroups was also non-significant for the primary outcome.

Aspirin reduced the rate of the two secondary composite outcomes: the rate of major vascular events was reduced by $34 \%(8.0 \%$ per year with placebo vs. $5.2 \%$ per year with aspirin; HR with aspirin, 0.66; $95 \%$ CI, 0.48-0.92; $P=0.01$ ) and the net clinical benefit (reduction in the rate of the myocardial infarction, stroke, venous thromboembolism, major bleeding, or death from any cause) was $33 \%$ in aspirin group, with an event rate of $9 \%$ per year in the placebo group and $6 \%$ per year in aspirin group (hazard ratio with aspirin, $0.67 ; 95 \% \mathrm{CI}, 0.49-0.91 ; P=0.01)$.

There were no significant differences between the two groups in the safety outcome (a rate of $0.6 \%$ events per year in the placebo group and $1.1 \%$ in the aspirin group, $P=0.22$ ).

The final meta-analysis, in which results from WARFASA and ASPIRE trial were combined, showed that aspirin significantly reduces the primary outcome (hazard ratio [HR] with aspirin, 0.68; $95 \%$ confidence interval [CI], 0.51-0.90; $P=0.007)$; a reduction of the secondary outcome (major vascular event) is also confirmed (hazard ratio [HR] with aspirin, 0.66; $95 \%$ confidence interval [CI], 0.51-0.86; $P=0.002$ ) without increasing bleedings (hazard ratio $[\mathrm{HR}]$ with aspirin, $1.47 ; 95 \%$ confidence interval [CI], 0.70-3.08; $P=0.31$ ).
Strengths of the study

- This is a well-designed study that addresses a relevant clinical issue, supported by a valid rationale.

- The primary endpoint is well defined and has a high clinical relevance; the follow-up period is adequate to evaluate VTE recurrences.

Weaknesses of the study

- The number of patients enrolled is severely lower than the sample size originally designed by the study protocol.

- The study is affected by a $22 \%$ combined rate of nonadherence to the study drug $(15 \%$ patients in the aspirin group discontinued the study drug and $7 \%$ in the placebo group initiated antiplatelet or anticoagulant treatment). Although the primary analysis has been done according to the intention-to-treat principle, we think that the high non-adherence rate might cause a bias in the study results.

Question marks

- About $25 \%$ patients in both groups had completed anticoagulant therapy for a period between 1 month and 2 years before randomization: it would be interesting to know if there is a difference in the average duration of this period between one group and the other.

- It is not clear how the protocols of the ASPIRE and WARFASA trials were prospectively harmonized to combine the results of the studies. We wonder if in the course of study any protocol changes were made, which could expose the study to a possible risk of bias.

- Patients with cancer do not meet the inclusion criteria, because they have an indication to anticoagulant therapy (with the exception of subjects at elevated risk of bleeding which is an exclusion criterion). We wonder why these patients were enrolled in the study.

\section{Sponsorship}

It is an independent study; Bayer provided aspirin and placebo but had no other role in the study.

\section{Clinical bottom line of the two studies}

In subjects with contraindications to assume anticoagulant therapy for more than 6 months, aspirin could be a valid therapeutic option for the prevention of venous thromboembolism recurrence. 


\section{Conflict of interest None.}

\section{References}

1. Kearon C, Akl EA, Comerota AJ (2012) Antithrombotic therapy for VTE disease: antithrombotic therapy and prevention of thrombosis, 9th edn: American College of Chest Physicians Evidence-Based Clinical Practice Guidelines. Chest 141:e419Se494S

2. Prandoni P, Lensing AW, Cogo A et al (1996) The long-term clinical course of acute deep venous thrombosis. Ann Intern Med 125:1-7

3. Antiplatelet Trialists' Collaboration (1994) Collaborative overview of randomised trials of antiplatelet therapy. III. Reduction in venous thrombosis and pulmonary embolism by antiplatelet prophylaxis among surgical and medical patients. BMJ 308:235-246

4. Members of the PEP Trial Collaborative Group (2000) Prevention of pulmonary embolism and deep vein thrombosis with low dose aspirin: pulmonary embolism prevention (PEP) trial. Lancet 355:1295-1302

5. Becattini C, Agnelli GA, Schenone A (2012) Aspirin for preventing the recurrence of venous thromboembolism. N Engl J Med 366:1959-1967

6. Brighton TA, Eikelboom JW, Mann K et al (2012) Low-dose aspirin for preventing recurrent venous thromboembolism. N Engl J Med 367:1979-1987 